\title{
Successful treatment of advanced gastric cancer by surgical resection following combination chemotherapy with oral S-1 and biweekly paclitaxel
}

\author{
Akiniro Nakajo, Shoji Natsugoe, Shuichi Hokita, Sumiya Ishigami, Hiroyuki Takatori, Takaaki Arigami, \\ Yoshikazu Uenosono, Kuniaki Aridome, and Takashi Aikou \\ Department of Digestive Surgery and Surgical Oncology, Kagoshima University Graduate School of Medical \& Dental Science, \\ 8-35-1 Sakuragaoka, Kagoshima 890-8520, Japan
}

\begin{abstract}
We report on the successful treatment of advanced gastric cancer by surgical resection following neoadjuvant chemotherapy. A 67-year-old man was referred to our hospital with a diagnosis of pancreatic cancer. Meticulous examination, however, revealed the presence of gastric cancer with ascites and large lymph node metastasis adjacent to the pancreas. We selected combination chemotherapy with oral S-1 and biweekly paclitaxel. After two courses, both the primary tumor and metastatic lymph nodes were greatly reduced, and the ascites had disappeared. Using laparoscopy, there was no evidence of peritoneal metastases, and the cytological examination was negative. The patient underwent distal gastrectomy with D2 lymph node dissection. Histological examination revealed that the cancer cells were still present in part, but no lymph node metastases were found. The tumor was pathologically diagnosed as pT2, pN0, P0, M0, CY0, and p-stage II. The patient is healthy over 4 years after surgery without recurrence.
\end{abstract}

Key words Chemotherapy $\cdot$ Paclitaxel $\cdot$ S-1 - Gastric cancer

\section{Introduction}

The detection of early-stage gastric cancer has led to an improved prognosis for patients with these tumors. However, the clinical outcome remains poor for patients with advanced gastric cancers, with peritoneal, multiple lymph node, or hematogenous metastases. The advent of new anticancer agents has enabled several novel types and regimens of chemotherapy to be tested in this patient group. S-1 (Taiho Pharmaceutical, Tokyo, Japan) is a new drug with single-agent response rates of reportedly more than $40 \%$ for advanced gastric cancer [1,2]. S-1 has also shown good efficacy in combination chemotherapy regimens. For example, the com-

Offprint requests to: A. Nakajo

Received: July 12, 2005 / Accepted: July 26, 2006 bination of S-1 and cisplatin (CDDP) showed response rates of more than $50 \%$ against advanced gastric cancer [3-8]. In clinical trials, CPT-11 + S-1 have shown similar high response rates of more than 50\% [9]. Preoperative combination chemotherapy of S-1 with CDDP, or other drugs, has been given to patients with unresectable tumors in an attempt to reduce the size of the primary tumor and the extent of the metastases with the hope of making surgery feasible [10].

Since 2001, we have investigated a new chemotherapy protocol based on the combination of S-1 and paclitaxel (Bristol-Myers Squibb, Tokyo, Japan). Paclitaxel inhibits cell replication by binding to and stabilizing microtubule polymers and induces mitotic arrest [11]. S-1 and paclitaxel have different mechanisms for their function and resistance as well as nonoverlapping toxicities [12]. We set up this combination chemotherapy of S-1 and biweekly paclitaxel and determined the maximum tolerated dose (MTD) and the dose limiting toxicity (DLT) of this regimen by a Phase I study in patients with advanced gastric cancer [13]. We report here on the successful treatment of a case of advanced gastric cancer by surgical resection following administration of oral S-1 combined with biweekly paclitaxel.

\section{Case report}

\section{Pretreatment diagnosis}

A 67-year-old man presented at his local hospital with epigastralgia. Following an abdominal ultrasonography (US) examination, he was initially diagnosed as having a pancreatic tumor and was referred to our specialist hospital. However, an abdominal computed tomography $(\mathrm{CT})$ scan showed thickening of the gastric wall and a bulky mass infiltrating the pancreas (Fig. 1A). Gastrointestinal endoscopy revealed a type 2 tumor measuring $7 \times 5 \mathrm{~cm}$ located on the gastric angle (Fig. $2 \mathrm{~A}$ ). 

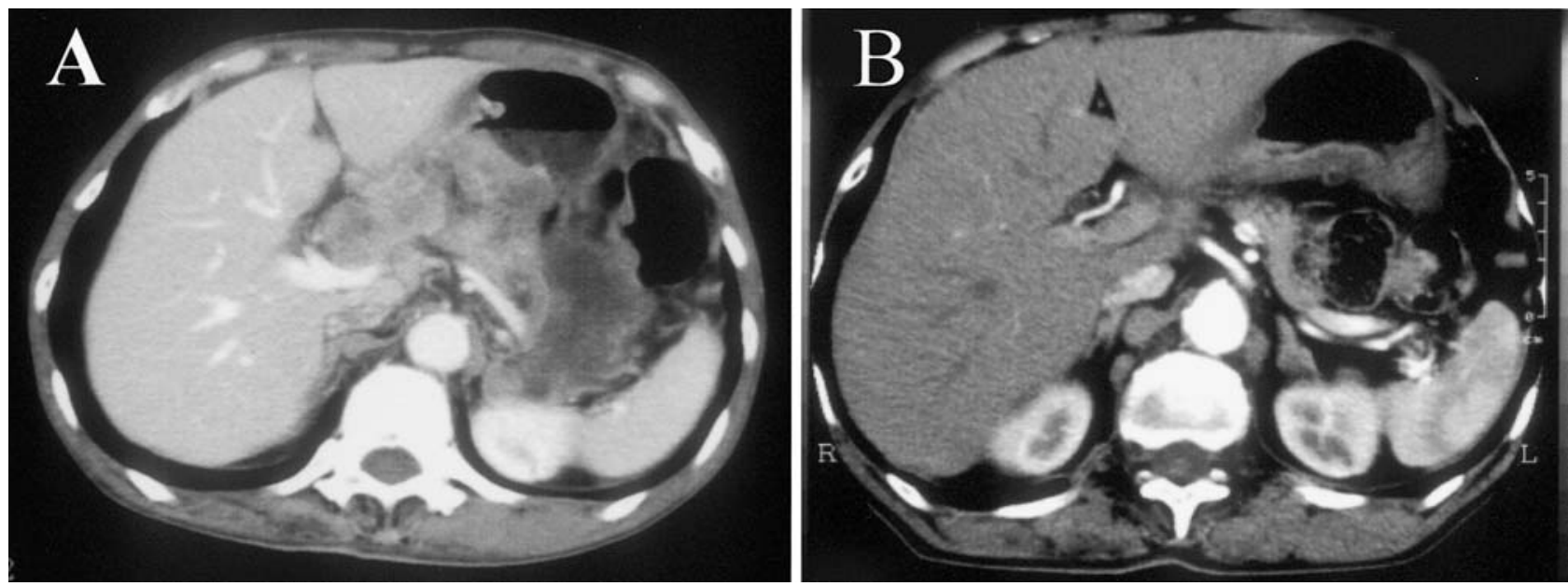

Fig. 1. A Abdominal computed tomography (CT) scans revealed the bulky lymph node metastasis extending mainly from the lesser curvature to the anterior common hepatic nodes. B CT scans after two courses of chemotherapy: the large metastatic lymph nodes have disappeared, and no ascites was found

Histological analysis of the biopsy specimen, removed during the endoscopy, identified a poorly differentiated adenocarcinoma. Therefore, the bulky mass adjacent to the pancreas, which had been seen on the CT scan, was diagnosed as a large lymph node metastasis from gastric cancer. Abdominal US also showed a large tumor with ascites, suggesting peritoneal dissemination. Regarding tumor markers, the serum carcinoembryonic antigen (CEA) level was $10.9 \mathrm{ng} / \mathrm{ml}$, and the carbohydrate antigen (CA) 19-9 level was $46.1 \mathrm{U} / \mathrm{ml}$. Based on the clinical findings and the Japanese classification of gastric carcinoma, the tumor was diagnosed as cT3(SE), cN2, H0, $\mathrm{P} 1, \mathrm{M} 0$, and c-stage IV [14]. Therefore, we decided that this tumor required chemotherapy.

\section{Chemotherapy}

Chemotherapy was performed using two anticancer agents, S-1 and paclitaxel. Oral S-1 $\left(80 \mathrm{mg} / \mathrm{m}^{2}\right)$ was administered twice a day after meals for 2 weeks; paclitaxel $\left(100 \mathrm{mg} / \mathrm{m}^{2}\right)$ was administered intravenously (i.v.) on days 1 and 15 only. The patient received two cycles of this regimen, separated by a 14-day interval. With respect to toxicity, the patient experienced grade 2 leukopenia and grade 1 nausea.

After the second course of chemotherapy, the response was assessed by imaging using endoscopy and CT scanning. Endoscopy revealed that the primary lesion was noticeably reduced, with shrinkage of the gastric wall (Fig. 2B). Based on the CT examination, the large metastatic lymph node had disappeared, and there were no signs of ascites (Fig. 1B). CEA and CA19-9 values had both decreased to within normal limits: $3.5 \mathrm{ng} / \mathrm{ml}$ and $21.2 \mathrm{U} / \mathrm{ml}$, respectively.

\section{Intraoperative findings}

Prior to laparotomy, intraabdominal observation was performed using a laparoscope. Because no overt peritoneal metastases were found and the cytological examination was negative, we judged that the tumor, including the lymph node metastases, was now resectable. Following chemotherapy, the primary lesion and large lymph node metastases were dramatically reduced. A distal gastrectomy was performed, with D2 lymph node dissection. A total of 44 lymph nodes were dissected. The Billroth I method was used for reconstruction of the alimentary tract.

\section{Histological findings}

Macroscopic examination of the resected specimen showed formation of an ulcer scar at the gastric angle (Fig. 3). Histological evidence showed that cancer cells were present, in part, in the mucosa and subserosa; and histological grading of chemotherapeutic efficacy was grade 2 (Fig. 4). Thirty regional nodes were dissected, but no lymph node metastases were found, including the large metastasis previously located adjacent to the pancreas. A pathological diagnosis was made of pT2, N0, P0, M0, CY0, and p-stage II.

\section{Postoperative course}

One week after the gastrectomy, the patient was reoperated on for an adhesional ileus. Thereafter, his postoperative course was uneventful, and he was discharged from hospital on day 35. The patient is followed up in the outpatient clinic without further chemotherapy. He is healthy over 4 years after surgery without recurrence. 

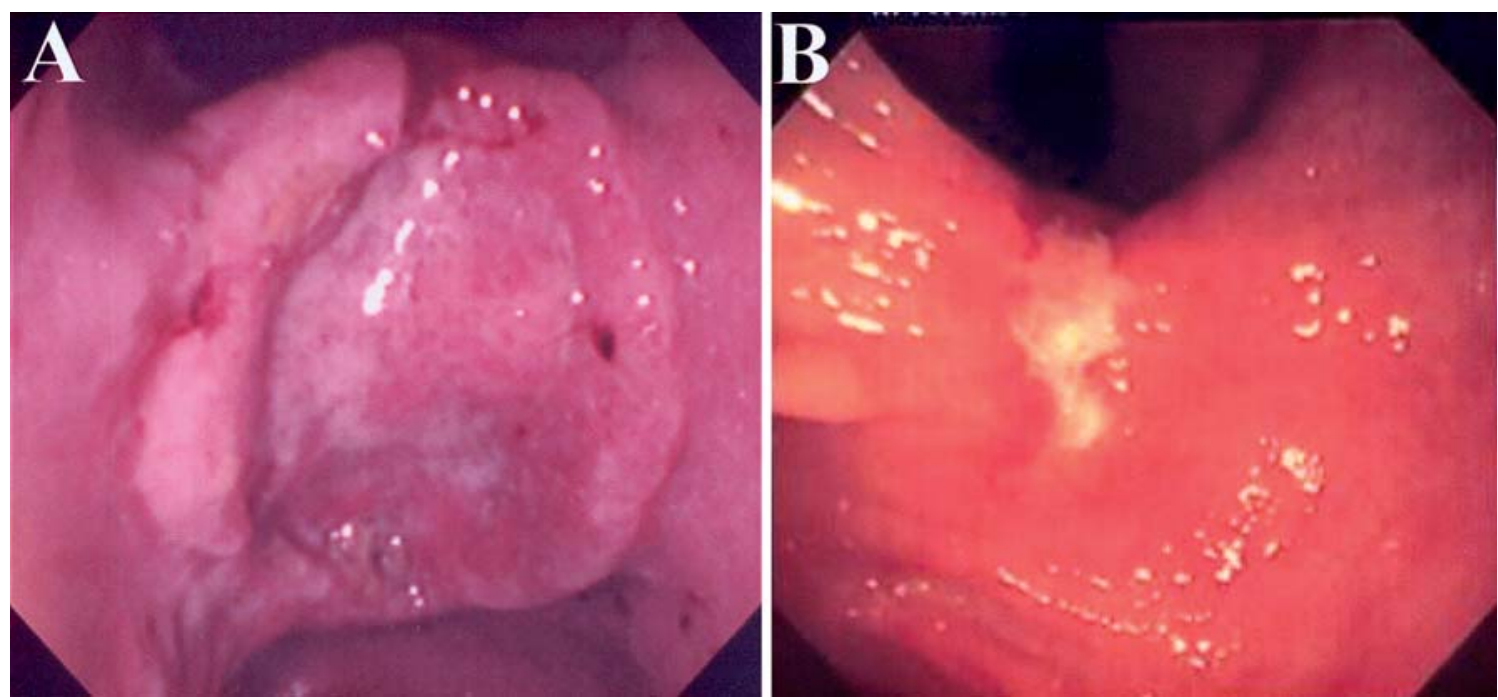

Fig. 2. A Gastrointestinal endoscopy revealed a type 2 tumor, measuring $7 \times 5 \mathrm{~cm}$, located in the gastric angle. B After two courses of chemotherapy, endoscopy revealed that the primary lesion was greatly reduced, with shrinkage of the gastric wall

\section{Discussion}

Recent reports have shown that S-1 is associated with good response rates when administered to patients with advanced and unresectable gastric cancer. The response rates of single-agent $\mathrm{S}-1$ have been reported at $40 \%-$ $50 \%[1,2]$. In some patients, distant metastases have disappeared after administration of single-agent S-1 [15]. Chemotherapy regimens have been devised that combined S-1 with other anticancer drugs, such as CDDP or paclitaxel; and these protocols have achieved clinical success [3-10]. In 2001, we started using a combination chemotherapy regimen of S-1 and paclitaxel in the treatment of unresectable gastric cancer.

The fluoropyrimidine S-1 is an oral combined form of tegafur (FT), a prodrug of 5-fluorouracil (5-FU), and two modulators, gimeracil (CDHP) and oteracil potassium (Oxo). It owes its antitumor properties to the production of 5-FU, which is gradually converted from FT following oral administration of S-1. S-1 is designed to enhance the clinical utility of an oral fluoropyrimidine but at the same time is associated with low gastrointestinal toxicity $[16,17]$. Paclitaxel binds with high affinity to microtubules and enhances tubulin polymerization. This action inhibits the normal dynamic process of the cell's microtubule network, resulting in the inhibition of mitosis and cell division [11]. The disappearance of peritoneal metastasis and the large primary tumor, which we observed in our patient, might be attributed to this inhibitory action.

The patient described in this case report had a large lymph node metastasis that was initially and mistakenly believed to be a pancreatic tumor. Furthermore, perito- neal dissemination was strongly suspected because of the presence of ascites. Even if the tumor and lymph node metastases could have been removed, the longterm survival of our patient was not expected. Therefore, we decided to attempt, first of all, to reduce the tumor load via chemotherapy. S-1 and paclitaxel are known to be associated with serious adverse effects, including bone marrow suppression, gastrointestinal symptoms, and alopecia [12,13]. However, our patient tolerated this combination chemotherapy regimen well, and no serious adverse effect occurred.

Following two courses of chemotherapy, imaging revealed a good response. However, because it was not possible to identify the presence or absence of a tiny nodule of peritoneal metastasis by imaging, laparoscopy was performed. A laparoscopic diagnosis is essential to determine if surgery is indicated following neoadjuvant treatment. Likewise, a cytological examination of lavage fluid is important when deciding if complete resection is feasible. Based on the laparoscopic findings in our patient, we decided that complete resection was possible.

One of the most important problems facing any clinician is when to perform surgery. In our case, a complete response might have been achieved if we had continued the chemotherapy. Histological examination showed residual cancer cells in parts of the mucosa and subserosa but no lymph node metastases. At present, however, we do not know whether such tiny lesions that were resistant to the initial chemotherapy would completely disappear after further courses. In the future, we should evaluate the timing of surgery after neoadjuvant chemotherapy by studying this issue in a large number 

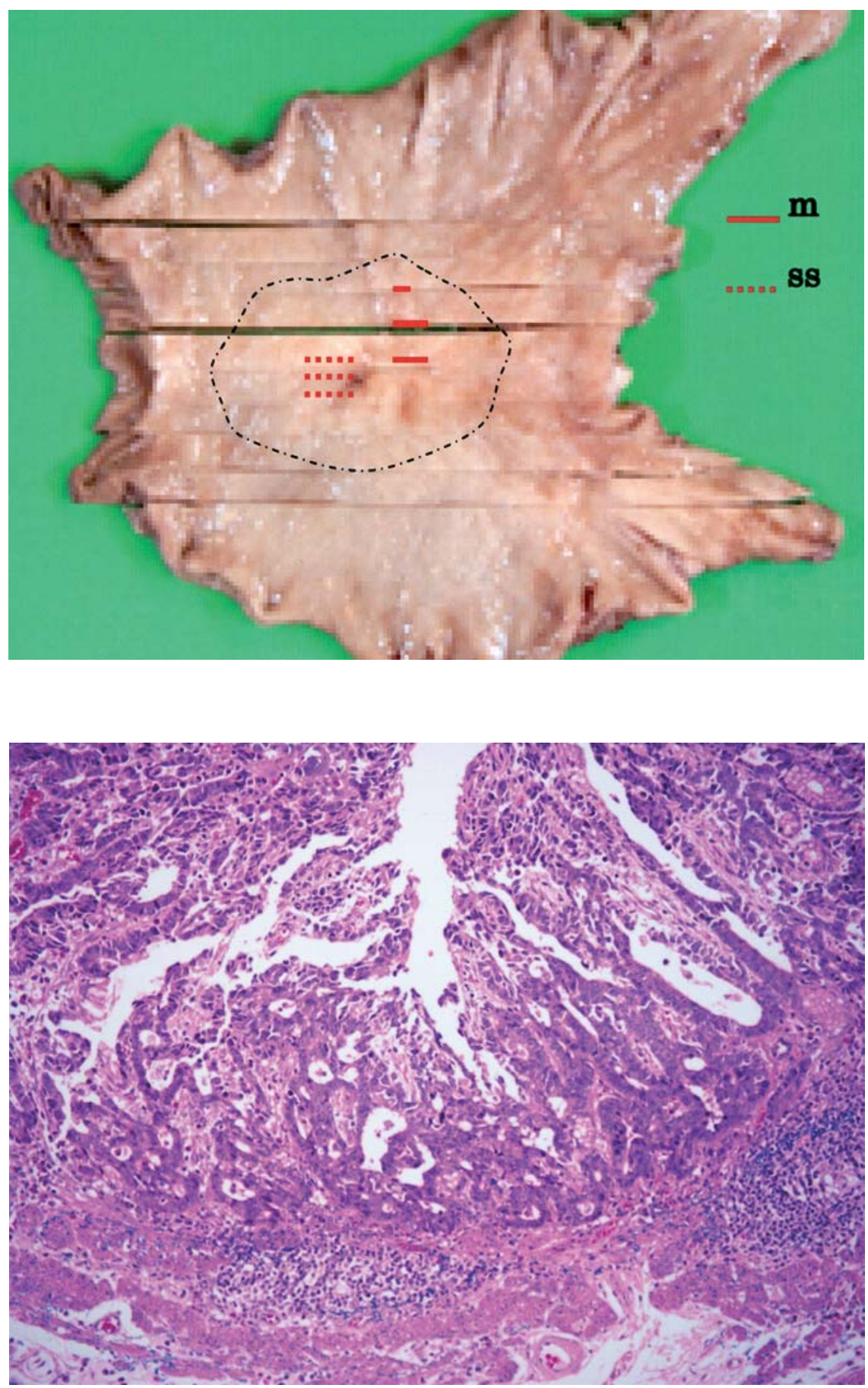

Fig. 3. Macroscopic examination of the resected specimen after chemotherapy shows ulcer scar formation in the gastric angle. The area surrounded by a dotted line shows the range of the primary lesion before chemotherapy. A few cancer cells were observed in a small area on a red line. $m$, mucosa; ss, subserosa
Fig. 4. Histological evidence of the partial presence of cancer cells in the mucosa and subserosa following chemotherapy and surgery. Histological grading of the chemotherapeutic efficacy was grade 2 of patients. Using chemotherapy followed by surgery benefited the long-term survival of our patient. We therefore conclude that the combined chemotherapy regimen of S-1 and paclitaxel is useful as a treatment for unresectable gastric cancer. However, randomized controlled studies are necessary to evaluate fully the effect of such chemotherapy.

\section{References}

1. Sakata Y, Ohtsu A, Horikoshi N, Sugimachi K, Mitachi Y, Taguchi T. Late phase II study of novel oral fluoropyrimidine anticancer drug S-1 (1 M tegafur- $0.4 \mathrm{M}$ gimestat-1 M otastat potassium) in advanced gastric cancer patients. Eur J Cancer 1998;34:1715-20.

2. Koizumi W, Kurihara M, Nakano S, Hasegawa K. Phase II study of S-1, a novel oral derivative of 5-fluorouracil, in advanced gastric cancer: for the S-1 Cooperative Gastric Cancer Study Group. Oncology 2000;58:191-7. 
3. Iwahashi M, Nakamori $M$, Tani M, Yamaue H, Sakaguchi S, Nakamura M, et al. Complete response of highly advanced gastric cancer with peritoneal dissemination after new combined chemotherapy of S-1 and low-dose cisplatin: report of a case. Oncology 2001;61:16-22.

4. Baba H, Yamamoto M, Endo K, Ikeda Y, Toh Y, Kohnoe S, et al. Clinical efficacy of S-1 combined with cisplatin for advanced gastric cancer. Gastric Cancer 2003;6:45-9.

5. Tsujitani S, Fukuda K, Kaibara N. Combination chemotherapy of S-1 and low-dose cisplatin for advanced gastric cancer. Gastric Cancer 2003;6:50-7.

6. Hyodo I, Nishina T, Moriwaki T, Endo S, Terao T, Hirao K, et al. A phase I study of S-1 combined with weekly cisplatin for metastatic gastric cancer in an outpatient setting. Eur J Cancer 2003; 39:2328-33.

7. Koizumi W, Tanabe S, Saigenji K, Ohtsu A, Boku N, Nagashima F, et al. Phase I/II study of S-1 combined with cisplatin in patients with advanced gastric cancer. Br J Cancer 2003;89:220712.

8. Sato Y, Kondo H, Honda K, Takahari D, Sumiyoshi T, Tsuji Y, et al. A phase I/II study of S-1 plus cisplatin in patients with advanced gastric cancer: 2 -week S-1 administration regimen. Int $\mathbf{J}$ Clin Oncol 2005;10:40-4.

9. Takahashi Y, Sakamoto J, Takeuchi T, Mai M, Kubota T, Kitajima M, et al. A randomized phase II clinical trial of tailored CPT-11 + S-1 vs S-1 in patients with advanced or recurrent gastric carcinoma as the first line chemotherapy. Jpn J Clin Oncol 2004;34:342-5.

10. Akatsu Y, Saikawa Y, Kubota T, Yoshida M, Furukawa T, Otani $\mathrm{Y}$, et al. Clinical and pathological disappearance of peritoneal dissemination in a patient with advanced gastric cancer receiving chemotherapy with S-1 and low-dose cisplatin. Gastric Cancer 2004; 7:128-33.

11. Crossin KL, Carney DH. Evidence that microtubule depolymerization early in the cell cycle is sufficient to initiate DNA synthesis. Cell 1981;23:61-71.

12. Schipper DL, Wagener DJT. Chemotherapy of gastric cancer. Anticancer Drugs 1996;7:137-49.

13. Hokita S, Aikou T, Miyazono F, Ishigami S, Aridome K, Maenohara S, et al. A phase I combination chemotherapy study of biweekly paclitaxel and S-1 administration in patients with advanced gastric cancer. Cancer Chemother Pharmacol 2005; $15: 1-5$

14. Japanese Gastric Cancer Association. Japanese classification of gastric carcinoma, 2nd English edition. Gastric Cancer 1998;1:10 24

15. Watanabe S, Tanaka T, Takeuchi T, Takabayashi H, Hirayama Y. Advanced gastric cancer with liver metastases successfully treated with S-1. Int J Clin Oncol 2002;7:326-9.

16. Shirasaka T, Shimamato Y, Ohshimo H, Yamaguchi M, Kato T, Yonekura $\mathrm{K}$, et al. Development of a novel form of an oral 5fluorouracil derivative (S-1) directed to the potentiation of the tumor selective cytotoxicity of 5-fluorouracil by two biochemical modulators. Anticancer Drugs 1996;7:548-57.

17. Takechi T, Nakano K, Uchida J, Mita A, Toko K, Takeda S, et al. Antitumor activity and low intestinal toxicity of S-1, a new formulation of oral tegafur, in experimental tumor models in rats. Cancer Chemother Pharmacol 1997;39:205-11. 